\title{
Rheumatoid Arthritis: An Exceptional Complication of Chemotherapy in a Patient with Lung Cancer
}

\author{
Tse-Hao Lee ${ }^{1}$, Rong-Hsin Yang ${ }^{1}$, Yum-Kung Chu ${ }^{1,2,3, *}$ \\ ${ }^{1}$ Department of Nuclear Medicine, Taipei Veterans General Hospital, Taiwan \\ ${ }^{2}$ National Yang-Ming University School of Medicine, Taiwan \\ ${ }^{3}$ National Defense Medical Center, Taipei, Taiwan \\ *Corresponding author: ykchu@vghtpe.gov.tw
}

Received March 19, 2015; Revised April 10, 2015; Accepted April 15, 2015

\begin{abstract}
A 63-year-old non-smoker, without previous history of arthritis, developed inflammatory arthritis while being treated with pemetrexed/cisplatin combination for advanced lung cancer. Clinical features and serology fulfilled the 2010 ACR/EULAR criteria for the diagnosis of rheumatoid arthritis (RA). RA is an autoimmune disease that primarily attacks joint tissues, causing inflammation of the joint lining. Although the relationship between these anticancer agents and the presence of RA remains unclear, this unprecedented adverse effect can be corrected by changing therapeutic strategies. Our case report might open a medical debate about chemotherapy side effect in cancer patients.
\end{abstract}

Keywords: anti-cyclic citrullinated peptide, autoimmunity, pemetrexed, rheumatoid arthritis

Cite This Article: Tse-Hao Lee, Rong-Hsin Yang, and Yum-Kung Chu, "Rheumatoid Arthritis: An Exceptional Complication of Chemotherapy in a Patient with Lung Cancer.” American Journal of Medical Case Reports, vol. 3, no. 5 (2015): 130-132. doi: 10.12691/ajmcr-3-5-3.

\section{Introduction}

Rheumatoid arthritis (RA) is an autoimmune disease, but there are no reported cases of chemotherapy-induced RA. We present herein the case of a 63-year-old woman with advanced lung cancer who developed painful swelling of joints after the first session of chemotherapy with pemetrexed/cisplatin combination. Clinical features and serology fulfilled the 2010 American College of Rheumatology (ACR) and European League Against Rheumatism (EULAR) classification criteria for the diagnosis of RA [1]. Her syndromes resolved after the off of chemotherapy and by the use of disease-modifying antirheumatic drug (DMARD).

\section{Case Report}

A 63-year-old woman presented with cough and 4-kg unintentional weight loss in recent four months. Her past history was non-contributory. She was a non-smoker without history of arthritis or diabetes. Chest plain film and computerized tomography (CT) revealed a heterogenous mass in the right lower lung, and multiple hypodense lesions in the liver. A diagnosis of adenocarcinoma of the lung was established based on the cytologic examination of bronchial lavage fluid. F-18 FDG PET/CT was referred for treatment planning and demonstrated avid lesions in the right lung and liver, confirming to the features of right lower lung carcinoma and metastatic deposits in right hilum and liver (Figure 1). The patient was diagnosed with clinical stage IV (T2a, N3, M1b) adenocarcinoma of the lung. She received six cycles of pemetrexed/cisplatin (Alimta ${ }^{\circledR} /$ Platamine ${ }^{\circledR}$ ). The induction was completed on $\mathrm{Jul} / 29 / 2013$ and chemotherapy was suspended for treatment assessment. The patient achieved a stable disease base on CT results and had no sign of bone metastasis on Tc-99m MDP scan (Figure 2A). Cisplatin was removed and pemetrexed was kept in a maintenance approach then. During the course of pemetrexed maintenance, the patient felt painful joint swelling, migrating over her left shoulder, elbows, hands and ankles. No Serological findings were positivity for rheumatoid factor (RF, 414.6 IU/ml), anti-cyclic citrullinated peptide (anti-CCP, $274 \mathrm{U} / \mathrm{mL}$ ), and antinuclear antibodies (ANA, x160), and rising of inflammatory markers such as erythrocyte sedimentation rate (ESR, $104 \mathrm{~mm} / \mathrm{h}$ ) and C-reactive protein (CRP, 3.08 $\mathrm{mg} / \mathrm{dl}$ ). The clinical characteristics and laboratory findings fulfilled the criteria for the diagnosis of early rheumatoid arthritis. Hydroxychloroquine (Plaquenil ${ }^{\circledR}$ ) $20 \mathrm{mg}$ daily was prescribed for her arthritis.

Surveillance bone scan in December 2013 revealed increased radioactivity in the right elbow, wrists, left ankle and small joints of hands, consistent with active arthritis (Figure 2B, arrows). On plain films there was mild soft tissue swelling and preserved joint space of bilateral wrists. No joint space narrowing or erosive change was seen in the elbows, hands, knees or ankles. After four cycles of pemetrexed continuation maintenance, the lesions in the lung and liver progressed. Treatment was therefore switched to erlotinib (Tarceva ${ }^{\circledR}$ ) 150mg daily, and led to a 
stable disease till June 2014. Her arthritis nearly resolved six months after the off of chemotherapy (Figure 2C). In the absence of other identifiable causes for arthropathy, it was regarded as chemotherapy induced RA, probably associated with the use of pemetrexed.

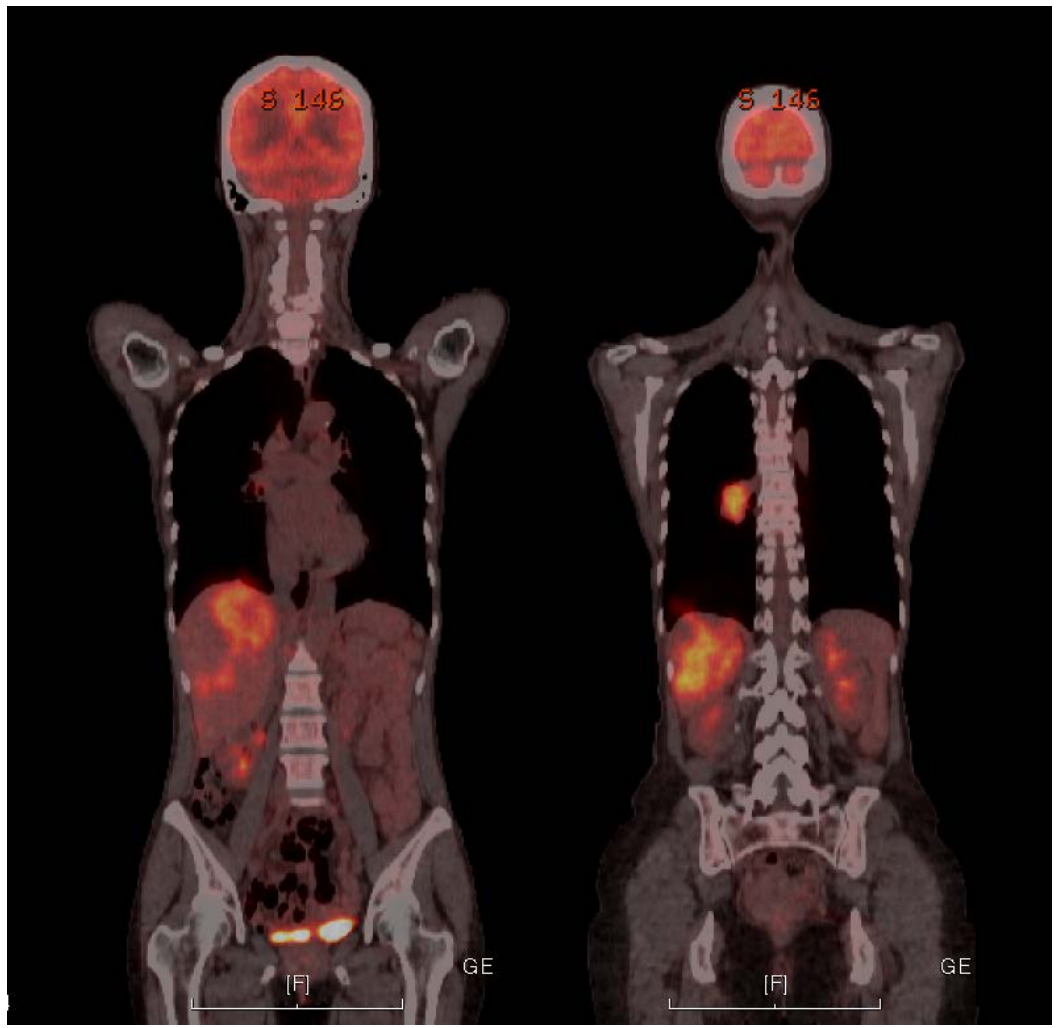

Figure 1. Coronal fused PET/CT displayed active lesions in the right lower lung with right paratracheal lymphadenopathy, and intrahepatic metastasis as seen by chest CT. Pemetrexed/cisplatin combination was used for advanced lung cancer

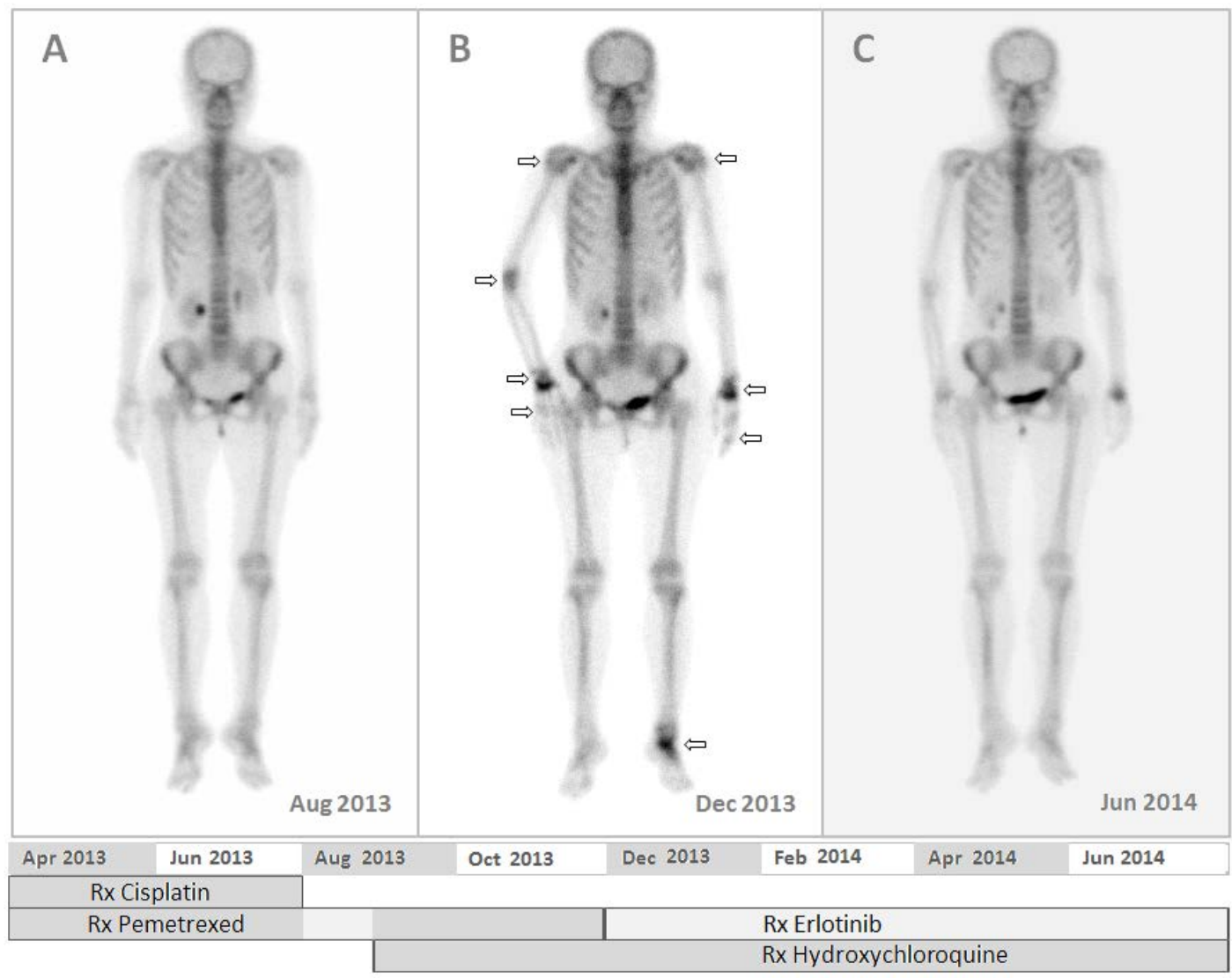

Figure 2. Series of surveillance bone scans. (A) At 4 months after completion of pemetrexed/cisplatin induction, bone scan showed no skeletal abnormality. (B) During the course of pemetrexed maintenance, the patient experienced arthralgia. Active arthritis was disclosed on bone scan. Clinical features and her seropositivity for RF and anti-CCP fulfilled the criteria for the diagnosis of RA. (C) Pemetrexed was switched to erlotinib targeted therapy. Hydroxychloroquine was also prescribed to treat arthropathy, which nearly resolved six months after the alternative strategy 


\section{Discussion}

The precise cause of RA is unknown. Genetic background and autoimmune factors are mainly responsible for the initiation of disease process [2]. RA is diagnosed clinically, and classified according to the 2010 ACR/EULAR classification criteria [1]. Local and systemic inflammation is one of the hallmarks of RA. Our patient with two or more swollen joints for 4 or more weeks, and her seropositivity for CRP, RF, ANA, and anti-CCP estimation, fulfilled the 2010 ACR/EULAR criteria for rheumatoid arthritis [1]. Early RA was defined by less than 12 months of symptoms according to the 1987 ACR criteria [3]. It is now well established that autoantibodies that are characteristic of RA, including RF and anti-CCP, are present before clinical disease onset [4]. $\mathrm{T}$ cells, B cells and the orchestrated interaction of proinflammatory cytokines play key roles in the pathophysiology of RA [5]. The early use of DMARDs has been recommended in recent years to reduce disease activity and prevent joint disability [1,6]. Both methotrexate and hydroxychloroquine are DMARDs. Hydroxychloroquine was prescribed in our patient and her arthropathy nearly resolved by six months.

Pemetrexed, analogous to methotrexate (MTX), blocks the folate-dependent steps in de novo purine and pyrimidine biosynthesis in diving cells and thereby stops cancer to spread and growth. Pemetrexed is approved for the treatment of advanced non-small-cell lung cancer as a combination partner of induction and a single maintenance agent [7]. Most chemotherapeutic drugs including folate antagonist work by impairing mitosis. Normal tissues with a high growth rate due to wear or tear are often harmed as the innocent bystanders. This affects actively growing cells such as those lining in the skin and intestine, blood cells, and the immune system. Observations of the microenvironment of rheumatoid joints revealed relevant events that in vitro, rheumatoid synovial fibroblasts grow at a faster rate and achieve a higher proliferative change [8].

MTX may reduce inflammation by suppressing the immune system. Actually low dose of MTX is currently regarded as the first medical treatment option for RA [9]. Interference with adenosine signaling as well as with nucleotide synthesis and cell proliferation probably contributed to the therapeutic effect [10]. MTX has been shown to induce apoptosis in fibroblast-like synovial cells (FLS) [11] and to inhibit FLS proliferation in vitro [12]. MTX is also used to treat other autoimmune diseases like multiple sclerosis [13], Crohn's disease [14], and lupus [15]. Lories et al. provided evidence that that MTX directly influence cell behavior, and reduced FLS proliferative activity. Mechanisms of MTX action, however, were more relevant in determining FLS growth rate [10].

Our patient developed RA, an unexpected autoimmune response, during the course of pemetrexed maintenance. Evidence to support autoimmune reaction is found in the fact that there is a rising of autoantibodies, namely RF and anti-CCP. In the absence of other identifiable causes for arthropathy, it was regarded that RA was related to anticancer regimens, probably pemetrexed. The authors speculate that the action of pemetrexed triggered the aberrant response, and led to active inflammation of joint tissues, on certain genetic background. The mechanism underlying arthrogenous toxicity has not yet been elucidated. This question may be resolved only by a prospective study of an anticancer population.

\section{Conclusion}

It is conceivable that chemotherapy triggered the aberrant response. Awareness of such unforeseen immunological reaction in cancer patients having chemotherapy by clinicians is crucial to excluding metastasis or other rheumatologic disorders, better in the early diagnosis, management or prevention.

\section{Statement of Competing Interests}

None of the authors have any competing interests.

\section{References}

[1] Aletaha D, Neogi T, Silman AJ, Funovits J, Felson DT, et al. 2010 rheumatoid arthritis classification criteria: an American College of Rheumatology/European League Against Rheumatism collaborative initiative. Arthritis Rheum 2010; 62;(9):2569-2581.

[2] Firestein GS. Etiology and pathogenesis of rheumatoid arthritis. In: Ruddy S, Harris E, Sledge C, eds. Kelly's Textbook of Rheumatology, 6th ed. Philadelphia: WB Saunders, 2001, 921-966.

[3] Arnett FC, Edworthy SM, Bloch DA, McShane DJ, Fries JF, et al. The American Rheumatism Association 1987 revised criteria for the classification of rheumatoid arthritis. Arthritis Rheum 1988; 31(3):315-324.

[4] Hitchon CA, El-Gabalawy HS. The synovium in rheumatoid arthritis. Open Rheumatol 2011; 5(suppl 1):107-114.

[5] Smolen JS, Steiner G. Therapeutic strategies for rheumatoid arthritis. Nat Rev Drug Discov 2003; 2(6):473-488.

[6] Saag KG, Teng GG, Patkar NM, Anuntiyo J, Finney C, Curtis JR, et al. American College of Rheumatology 2008 recommendations for the use of nonbiologic and biologic disease-modifying antirheumatic drugs in rheumatoid arthritis. Arthritis and rheumatism 2008; 59(6):762-84.

[7] Okamoto I, Aoe K, Kato T, Hosomi Y, Yokoyama A, et al. Pemetrexed and carboplatin followed by pemetrexed maintenance therapy in chemo-naive patients with advanced nonsquamous nonsmall-cell lung cancer. Invest New Drugs 2013; 31(5): 1275-1282.

[8] Bucala R, Ritchlin C, Winchester R, Ceram A. Constitutive production of inflammatory and mitogenic cytokines by rheumatoid synovial fibroblasts. J Exp Med 1991; 173(3): 569-574.

[9] Van der Heijden JW, Oerlemans R, Dijkmans BA, Qi H, van der Laken CJ, et al. Folate receptor beta as a potential delivery route for novel folate antagonists to macrophages in the synovial tissue of rheumatoid arthritis patients. Arthritis Rheum 2009; 60(1):1221.

[10] Lories RJU, Derese I, De Bari C, Luyten FP. In vitro growth rate of fibroblast-like synovial cells is reduced by methotrexate treatment. Ann Rheum Dis 2003; 62(6):568-571.

[11] Nakazawa F, Matsuno H, Yudoh K, Katayama R, Sawai T, et al. Methotrexate inhibits rheumatoid synovitis by inducing apoptosis. J Rheumatol 2001; 28(8):1800-1808.

[12] Meyer FA, Yaron I, Mashiah V, Yaron M. Methotrexate inhibits proliferation but not interleukin 1 stimulated secretory activities of cultured human synovial fibroblasts. J Rheumatol 1993; 20(2):238-242.

[13] Gray O, McDonnell GV, Forbes RB. Methotrexate for multiple sclerosis. Cochrane Database Syst Rev 2004 Apr 19; (2): CD003208. Review.

[14] McDonald JW, Tsoulis DJ, Macdonald JK, Feagan BG. Methotrexate for induction of remission in refractory Crohn's disease. Cochrane Database Syst Rev 2012 Dec 12; (12): CD003459.

[15] Wong JM, Esdaile JM. Methotrexate in systemic lupus erythematosus. Lupus 2005; 14(2), 101-105. 